\title{
Submitting to an OJS Journal
}

See the video here: https://www.youtube.com/watch?v=Eg0N8ljT6AY 